\title{
Robust Control of Magnetic Bearing in the Inertially Stabilized Platform for Aerial Remote Sensing
}

\author{
Qingyuan Guo, Gang Liu and Tong Wen \\ School of Instrument Science and Optoelectronics Engineering, Beihang University, Beijing 100191, China
}

\begin{abstract}
Comparing with the normal mechanical inertial stabilized platform (ISP), the novel ISP with magnetic bearing to suspend the yaw gimballing owns the ability of isolating the vibration and disturbance of external gimballing. However, on the one hand, complex structure of the magnetically suspended gimballing introduces the parametric uncertainties into modelling of magnetic suspension force, and the disturbance between the magnetic fields brings the un-modeled force into dynamics of magnetically suspended gimballing. On the other hand, the external disturbances such as wind drag affect the stability of magnetically suspended gimballing. Therefore, to weaken the negative influence on stability and improve the robustness of system, the ${ }^{H_{\infty}}$ robust controllers for translation and tilting of gimballing are designed. The experimental results indicate that the robust controller of magnetically suspended gimballing owns the excellent robustness when the load of magnetically suspended gimballing changes.
\end{abstract}

\section{Introduction}

The ISP for remote sensing is promising to be applied in the observation and photography system fixed on the airborne platform and ship-based platform [1][2]. Usually, the loads (include POS and camera) are installed on the yaw gimballing, so stability and control precision of yaw gimballing are critical to the quality of image achieved by camera [3]. For the ordinary mechanical ISP with three axis frames, the motions of roll angle, pitch angle and inner yaw angle are separated by the mechanical levitation methods such mechanical bearing and gear[4][5]. However, for the mechanical levitation methods between gimballing, which is touched between the external gimballing and internal gimballing, the disturbance of external gimballing possibly transfer from the external gimballing to internal gimballing, so the control precision and stability of yaw gimballing are affected. Therefore, one kind of novel ISP with magnetic suspension technology was designed [6-8], the magnetic suspension method is untouched so that the disturbance can be effectively isolated. Furthermore, to provide a stable environment to the yaw gimballing, there were a series of relevant research to improve the robustness of magnetically suspended system, a gain scheduled $H_{\infty}$ robust control scheme with free parameters was proposed to eliminate the unbalance vibration in a rotor system supported by magnetic bearings [9]. And, for an active radial homopolar magnetic bearing system, the robust control of rigid rotor vibration was designed analytically, and the initial responses/transient responses and robustness of the designed controller was confirmed [10]. In addition, aiming at the difficulty of obtaining the accurate model of magnetic bearing, the robust stabilization of a voltage-controlled three pole active magnetic bearing was considered [11]. However, those papers only focused on control of the magnetically suspended rotor with high-speed and light-weight, and the magnetically suspended rotor did not need to bear load. There is no publication about magnetically suspended gimballing with great rotary inertia, great bearing ability and wide load range until now. Therefore, in this paper, considering the parametric uncertainties, external disturbance and change of load, the robust control of magnetically suspended gimballing is designed, and the suspension performance under the situations of different loads is tested.

\section{Model of the magnetically suspended gimballing}
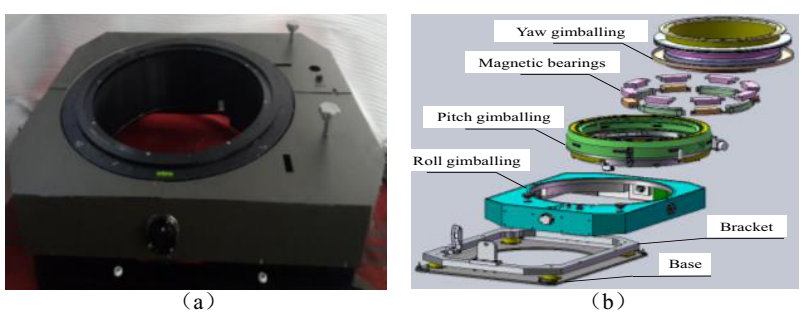

Figure 1. Structure of ISP with magnetic bearings. (a) photograph, (b) exploded view. 
The configuration of ISP with magnetic bearings is shown as Fig.1, the whole ISP consists of three gimballing---roll gimballing, pitch gimballing and yaw gimballing. And, yaw gimballing, as the innermost gimballing, all load components are installed on it, its control precision and stability are critical to the quality of imaging. In this paper, magnetic bearings, instead of mechanic bearings and gears, are applied to levitate the yaw gimballing in order to absolutely isolate disturbance from outer gimballing. For the pitch gimballing, it is outsider the yaw gimballing, the roll gimballing is the outermost gimballing.

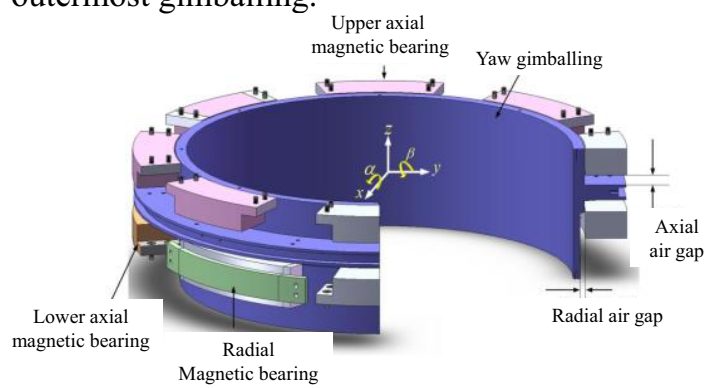

Figure 2. Structure of magnetically suspended gimballing.

As illustrated as Fig.2, the yaw gimballing is suspended by magnetic bearings in radial and axial directions, the structure of magnetic suspended gimballing is composed with radial magnetic bearing and axial magnetic bearing. Eight same axial magnetic bearings are located at the upper surface of yaw gimballing, four same axial magnetic bearings are fixed on lower surface, which are mounted on circumferential position as the four of upper axial magnetic bearings to control the axial translation. And, other four magnetic bearings control the tilting of gimballing around $\mathrm{X}$ and $\mathrm{Y}$ axis. In addition, radial translation of magnetically suspended gimballing is controlled by two pairs of magnetic bearings which are installed in radial direction. As a consequent, the suspension of yaw gimballing on five degree of freedom is realized.

\subsection{The translational model of magnetically suspended gimballing}

Virtually, the translational control of the magnetically suspended gimballing is the control of magnetic bearing in radial direction and axial direction, the position and velocity of inner yaw gimballing are controlled by adjusting the force generated by magnetic bearing.
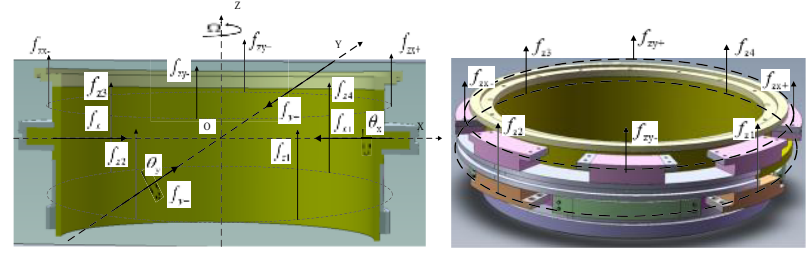

Figure 3. Analysis of force received by magnetically suspended gimballing.

The radial control of magnetically suspended gimballing is to realize the stable suspension, according to the analysis in Fig.3, the dynamic model of magnetically suspended gimballing in the radial and axial directions is

$\left\{\begin{array}{l}m \ddot{x}=f_{\mathrm{x}} \\ m \ddot{y}=f_{y} \\ m \ddot{z}=f_{z}\end{array}\right.$

(1)

The translational model of magnetically suspended gimballing is expressed as the transfer function of

$$
\left\{\begin{array}{l}
G_{\mathrm{x}}(s)=\frac{X(s)}{I_{\mathrm{x}}(s)}=\frac{k_{\mathrm{ix}}}{m s^{2}-k_{\mathrm{dx}}} \\
G_{\mathrm{y}}(s)=\frac{Y(s)}{I_{\mathrm{y}}(s)}=\frac{k_{\mathrm{iy}}}{m s^{2}-k_{\mathrm{dy}}} \\
G_{\mathrm{z}}(s)=\frac{Z(s)}{I_{\mathrm{z}}(s)}=\frac{k_{\mathrm{iz}}}{m s^{2}-k_{\mathrm{dz}}}
\end{array}\right.
$$

Furthermore, the state variable of model is selected as $X_{\mathrm{p}}=\left(\begin{array}{llllll}x_{\mathrm{p}} & y_{\mathrm{p}} & z_{\mathrm{p}} & \dot{x}_{\mathrm{p}} & \dot{y}_{\mathrm{p}} & \dot{z}_{\mathrm{p}}\end{array}\right)^{\mathrm{T}}$, input variable is $U_{\mathrm{p}}=\left(\begin{array}{lll}i_{\mathrm{x}} & i_{\mathrm{y}} & i_{z}\end{array}\right)^{\mathrm{T}}$

\section{.}

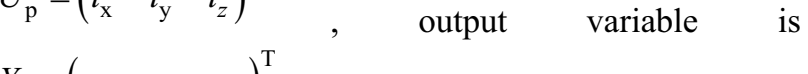
$Y_{\mathrm{p}}=\left(\begin{array}{lll}x_{\mathrm{p}} & y_{\mathrm{p}} & z_{\mathrm{p}}\end{array}\right)^{\mathrm{T}}$, and state space function of translational system is

$\dot{X}_{\mathrm{p}}=A_{\mathrm{p}} X_{\mathrm{p}}+B_{\mathrm{p}} U_{\mathrm{p}}$

And the output function of translational system is

$Y_{\mathrm{p}}=C_{\mathrm{p}} X_{\mathrm{p}}+D_{\mathrm{p}} U_{\mathrm{p}}$

$$
\begin{aligned}
& A_{\mathrm{p}}=\left[\begin{array}{cccccc}
0 & 0 & 0 & 1 & 0 & 0 \\
0 & 0 & 0 & 0 & 1 & 0 \\
0 & 0 & 0 & 0 & 0 & 1 \\
\frac{k_{\mathrm{dx}}}{m} & 0 & 0 & 0 & 0 & 0 \\
0 & \frac{k_{\mathrm{dy}}}{m} & 0 & 0 & 0 & 0 \\
0 & 0 & \frac{k_{\mathrm{d} z}}{m} & 0 & 0 & 0
\end{array}\right], B_{\mathrm{p}}=\left[\begin{array}{ccc}
\frac{k_{\mathrm{ix}}}{m} & 0 & 0 \\
0 & \frac{k_{\mathrm{iy}}}{m} & 0 \\
0 & 0 & \frac{k_{\mathrm{iz}}}{m}
\end{array}\right], \\
& \text { where } \\
& C_{\mathrm{p}}=\left[\begin{array}{llllll}
1 & 0 & 0 & 0 & 0 & 0 \\
0 & 1 & 0 & 0 & 0 & 0 \\
0 & 0 & 1 & 0 & 0 & 0
\end{array}\right], D_{\mathrm{p}}=\left[\begin{array}{lll}
0 & 0 & 0 \\
0 & 0 & 0 \\
0 & 0 & 0
\end{array}\right] .
\end{aligned}
$$

\section{The robust control of magnetically suspended gimballing}

\subsection{Robust control}

Captions should be typed in 9-point Times. They should be centred above the tables and flush left beneath the figures.

The robust control directly addresses the robustness of control system by designing robust controller, the standard $H_{\infty}$ robust control diagram is showed as the Fig.4, 


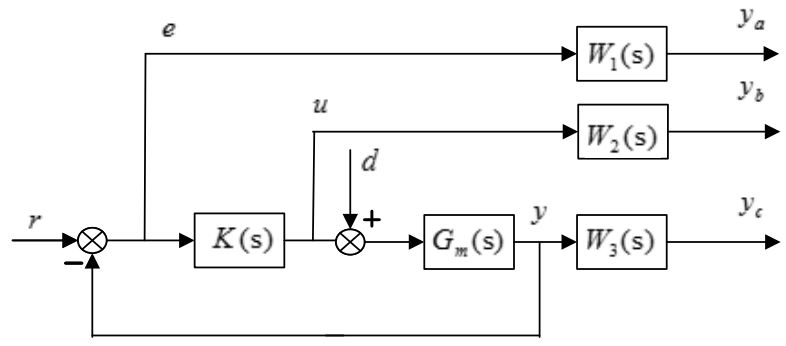

Figure 4. Robust control diagram.

where $r$ is the reference input signal, $e$ is the tracking error, $u$ the control input, $d$ measurement disturbance, $y$ the output of system, $W_{1}(s)$ is the performance weighting function, $W_{2}(s)$ is the input weighting function and $W_{3}(s)$ is the output weighting function.

Given $G_{\mathrm{m}}(\infty)=0$, the closed-loop transfer function from $r$ to $e, u$ and $y$ is, respectively

$S=\frac{E(s)}{R(s)}=\frac{1}{1+G_{\mathrm{m}}(s) K(s)}$

$R=\frac{U(s)}{R(s)}=\frac{K(s)}{1+G_{\mathrm{m}}(s) K(s)}$

$T=\frac{Y(s)}{R(s)}=\frac{G_{\mathrm{m}}(s) K(s)}{1+G_{\mathrm{m}}(s) K(s)}$

where $S$ is the sensitivity function, $R$ is the penalty sensitivity function, and $S+R=I$.

During the procedure of designing suboptimal $H_{\infty}$ robust controller, we try to find a stabilizing controller $K(s)$, which ensure the closed-loop system stable, and $\|P\|_{\infty}$, the closed-loop transfer function of enhanced object model, must satisfy

$\min \|P\|_{\infty}=\min \left\|\begin{array}{l}W_{1}(s) S \\ W_{2}(s) R \\ W_{3}(s) T\end{array}\right\|_{\infty}=\gamma_{0} \leq\|P\|_{\infty}=\gamma$

where $\gamma$ is a minimal value of the optimization factor, and smaller $\gamma$ is, the stronger the robustness.

\subsection{Design of weighting matrix}

$A$, The selection of $W_{1}(s)$

For the magnetic suspended gimballing, the frequency of disturbance usually locates at the low frequency domain, so the gain of $S$ should be small to inhibit the disturbance. Consequently, the weighting value should be high, $W_{1}(s)$ owns the characteristics of low-pass filter [12].

$B$, The selection of $W_{2}(s)$

$W_{2}(s)$ is usually selected as constant to decrease orders of controller.
$C$, The selection of $W_{3}(s)$

$W_{3}(s)$ expresses the norm bound of multiplicative perturbation, it is selected as the function which own the characteristic of high-pass filter, and the rising slope should be high. Therefore, the gain of $S$ would be decreased in the low frequency domain, and the gain of $T$ would be inclined in the high frequency domain.

In our case, the nominal function of magnetically suspended gimballing in radial direction ( $\mathrm{X}$ and $\mathrm{Y}$ axis) and axial direction ( $\mathrm{Z}$ axis) is respectively,

$G_{\mathrm{r} 0}(s)=G_{\mathrm{x}}(s)=G_{\mathrm{y}}(s)=\frac{300}{23 s^{2}-480000}$

$G_{\mathrm{a} 0}(s)=G_{\mathrm{z}}(s)=\frac{2000}{23 s^{2}-3000000}$

And, the nominal tilting function of magnetically suspended gimballing is

$G_{\mathrm{t} 0}(s)=G_{\mathrm{zx}}(s)=G_{\mathrm{zy}}(s)=\frac{2000}{12 s^{2}-3000000}$

Based on the selection principle of weighting function, taking the radial translation of magnetically suspended gimballing as example, its weighting functions are selected as

$\left\{\begin{array}{l}W_{\mathrm{r} 1}(s)=\frac{0.005 s+1010000}{s+10} \\ W_{\mathrm{r} 2}(s)=\frac{0.0001 s+5}{0.01 s+100000} \\ W_{\mathrm{r} 3}(s)=\frac{s+500}{0.01 s+1000}\end{array}\right.$

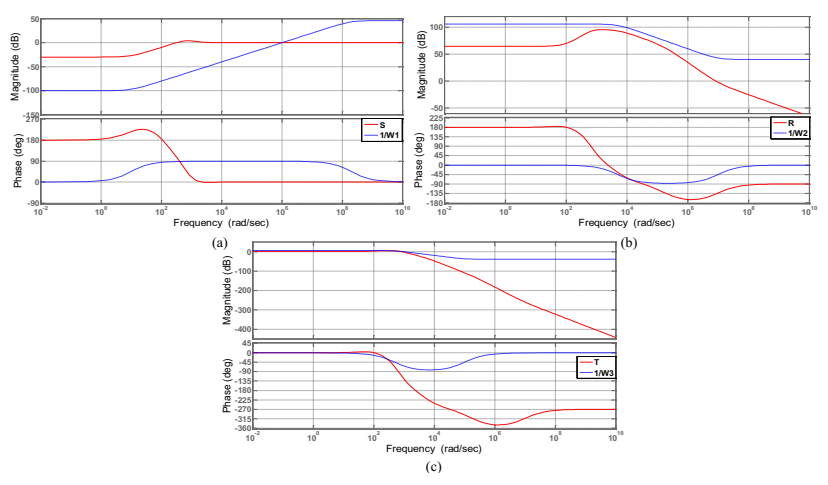

Figure 5. Characteristics of Weighing functions. (a) ${ }^{W_{\mathrm{r} 1}}(s)$, (b) $W_{\mathrm{r} 2}(s),(\mathrm{c}) W_{\mathrm{r} 3}(s)$

And the amplitude-frequency characteristics and phase-frequency characteristics of weighting functions $W_{\mathrm{r} 1}(s), W_{\mathrm{r} 2}(s)$ and $W_{\mathrm{r} 3}(s)$ are shown in Fig.5, and transfer function of the $H_{\infty}$ robust controller is

$K_{\mathrm{r}}(s)=\frac{5.492 \times 10^{6} s^{4}+5.547 \times 10^{13} s^{3}+5.505 \times 10^{18} s^{2}+1.255 \times 10^{21} s+6.664 \times 10^{22}}{s^{5}+2.792 \times 10^{5} s^{4}+1.901 \times 10^{10} s^{3}+1.112 \times 10^{14} s^{2}+1.281 \times 10^{17} s+1.27 \times 10^{18}}$ (13)

Similarly, for the axial translation of magnetically suspended gimballing, the weighting functions of axial translation are chosen as following, 
$\left\{\begin{array}{l}W_{\mathrm{a} 1}(s)=\frac{0.001 s+100200}{2 s+10} \\ W_{\mathrm{a} 2}(s)=\frac{0.0003 s+1.5}{0.01 s+100000} \\ W_{\mathrm{a} 3}(s)=\frac{2 s+700}{0.03 s+1200}\end{array}\right.$

(14)

And the transfer function of the $H_{\infty}$ robust controller is

$K_{\mathrm{a}}(s)=\frac{9.175 \times 10^{5} s^{4}+9.212 \times 10^{12} s^{3}+3.708 \times 10^{17} s^{2}+1.541 \times 10^{20} s+7.716 \times 10^{21}}{s^{5}+2.114 \times 10^{5} s^{4}+8.054 \times 10^{9} s^{3}+4.449 \times 10^{13} s^{2}+5.247 \times 10^{16} s+2.613 \times 10^{17}}$ (15)

In addition, for the tilting function of magnetically suspended gimballing, the weighting functions are chosen as

$$
\left\{\begin{array}{l}
W_{\mathrm{t} 1}(s)=\frac{0.001 s+1010200}{s+10} \\
W_{\mathrm{t} 2}(s)=\frac{0.0001 s+1.2}{0.01 s+100000} \\
W_{\mathrm{t} 3}(s)=\frac{3 s+800}{0.001 s+10000}
\end{array}\right.
$$

The transfer function of the $H_{\infty}$ robust controller is selected as following,

$$
K_{\mathrm{t}}(s)=\frac{3.273 \times 10^{6} s^{4}+6.546 \times 10^{13} s^{3}+3.274 \times 10^{20} s^{2}+2.383 \times 10^{23} s+3.73 \times 10^{25}}{s^{5}+1.023 \times 10^{7} s^{4}+2.274 \times 10^{12} s^{3}+1.923 \times 10^{16} s^{2}+5.467 \times 10^{19} s+5.447 \times 10^{20}}
$$

\section{Experimental results}

\subsection{Experimental setup}

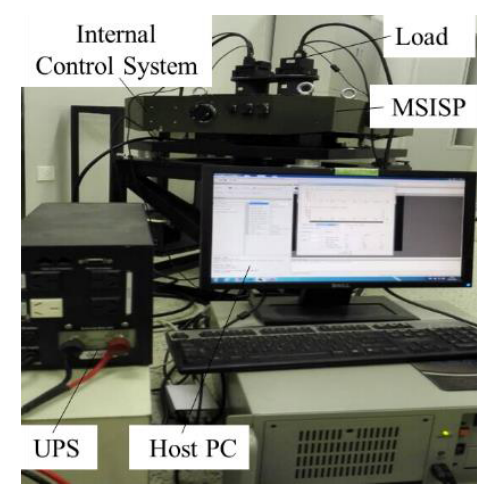

Figure 6. Experimental setup.

The experimental setup of magnetically suspended ISP is shown in Fig.6, the whole control circuit is embedded inside of magnetically suspended gimballing, and it consists of a digital signal processor TMS320F28335 with 12-bit A/D converter to control magnetic bearing, the digital PWM amplifier with $20 \mathrm{KHz}$ to drive the magnetic bearing, eddy current sensors to measure the displacement of rotor. In addition, the sampling frequency of whole control system is $20 \mathrm{KHz}$, the power supply provided by UPS is $28 \mathrm{~V}$, other system parameters of magnetically suspended gimballing are listed in the Table.1.

Table 1. System parameters of magnetically suspend gimballing

\begin{tabular}{cccc}
\hline Symbol & Value & Symbol & Value \\
\hline$k_{\mathrm{ix}}$ & $300 \mathrm{~N} / \mathrm{A}$ & $k_{\mathrm{dx}}$ & $480 \mathrm{~N} / \mathrm{mm}$ \\
$k_{\mathrm{iy}}$ & $300 \mathrm{~N} / \mathrm{A}$ & $k_{\mathrm{dy}}$ & $480 \mathrm{~N} / \mathrm{mm}$ \\
$k_{\mathrm{iz}}$ & $2000 \mathrm{~N} / \mathrm{A}$ & $k_{\mathrm{dz}}$ & $3000 \mathrm{~N} / \mathrm{mm}$ \\
$J_{\mathrm{x}}$ & $6.25 \mathrm{~N} \cdot \mathrm{m}^{2}$ & $J_{\mathrm{y}}$ & $6.25 \mathrm{~N} \cdot \mathrm{m}^{2}$ \\
$m$ & $23 \mathrm{~kg}$ & $l$ & $520 \mathrm{~mm}$ \\
\hline
\end{tabular}

\subsection{Suspension performance in translational direction}

When the load of magnetically suspended gimballing are $15 \mathrm{~kg}, 30 \mathrm{~kg}$ and $45 \mathrm{~kg}$, the axial suspension performance of magnetically suspended gimballing is tested. As shown as Fig.7, the red dashed line illustrates the displacement of PID controller, the blue line shows the displacement of robust controller. Initially, there is no load installed on the magnetically suspended gimballing, the gimballing stably suspend at the axial equilibrium point.
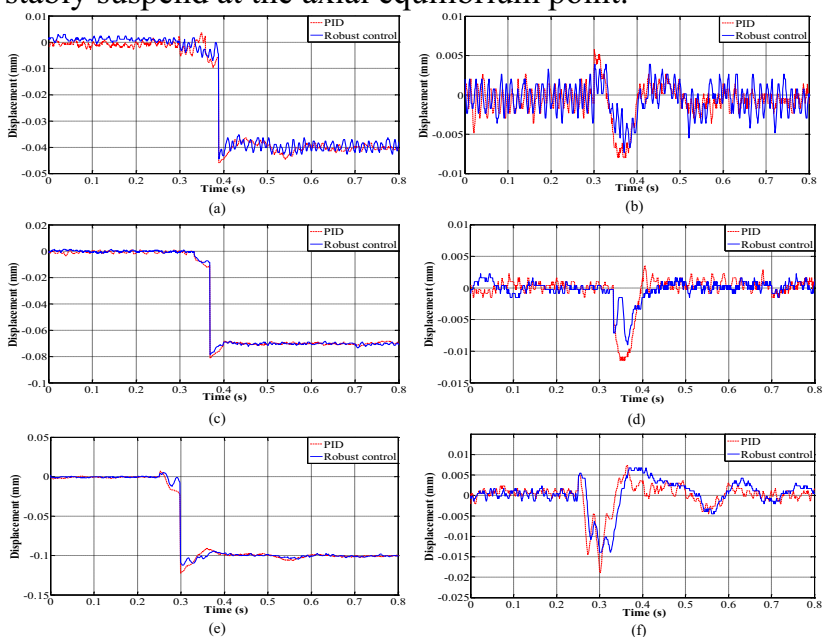

Figure 7.Translational suspension performance with loads, (a) axial suspension with $15 \mathrm{~kg}$, (b) radial suspension with $15 \mathrm{~kg}$, (c) axial suspension with $30 \mathrm{~kg}$, (d) radial suspension with $30 \mathrm{~kg}$, (e) axial suspension with $45 \mathrm{~kg}$, (f) radial suspension with $45 \mathrm{~kg}$.

Then, the different loads are installed on the magnetically suspended gimballing, the comparison of radial drift between the robust control and PID is presented in the Table.2, the axial overshoot comparison is illustrated in Table.3. Thus, it is concluded that displacement overshoot of robust control is quite smaller than that of PID control when the loads are different.

Table 2. Maximum radial drift comparison between PID and robust control

\begin{tabular}{cccc}
\hline & $\mathbf{1 5} \mathbf{k g}$ & $\mathbf{3 0 k g}$ & $\mathbf{4 5} \mathrm{kg}$ \\
\hline PID & $0.0075 \mathrm{~mm}$ & $0.012 \mathrm{~mm}$ & $0.02 \mathrm{~mm}$ \\
Robust Control & $0.006 \mathrm{~mm}$ & $0.009 \mathrm{~mm}$ & $0.012 \mathrm{~mm}$ \\
\hline
\end{tabular}


Table 3. Maximum axial overshoot comparison between PID and robust control

\begin{tabular}{cccc}
\hline & $15 \mathrm{~kg}$ & $\mathbf{3 0 k g}$ & $\mathbf{4 5 k g}$ \\
\hline PID & $0.043 \mathrm{~mm}$ & $0.076 \mathrm{~mm}$ & $0.11 \mathrm{~mm}$ \\
Robust Control & $0.046 \mathrm{~mm}$ & $0.082 \mathrm{~mm}$ & $0.12 \mathrm{~mm}$ \\
\hline
\end{tabular}

\section{Conclusion}

In this paper, we have analyzed the characteristics of magnetically suspended gimballing, the magnetically suspended gimballing owns greater bearing ability and wider bearing range, and the load of magnetically suspended gimballing will change according to the requirements. Consequently, those requirements introduce parametric uncertainties (unmodeled force, unmodeled dynamics), and the change of load cause a robustness problem to the magnetically suspended gimballing. Therefore, robust controllers of translation and tilting are designed to improve the robustness of magnetically suspended gimballing system. The experimental results demonstrate that robust controller has strong robustness when the load of the magnetically suspended gimballing changes.

\section{Acknowledgement}

This work is supported by the National Natural Science Foundation of China under Grant 61374211.

\section{References}

1. Hurak, Z. and M. Rezac. Image-based pointing and tracking for inertially stabilized airborne camera platform. IEEE Transactions on Control Systems Technology; 20(5): 1146-1159(2012).

2. Moon, J. and S.Y. Jung. Implementation of an image stabilization system for a small digital camera. IEEE Transactions on Consumer Electronics; 54(2): 206-212. (2008)

3. Mokbel, H.F., et al. Modeling and optimization of Electro-Optical dual axis Inertially Stabilized Platform. in Optoelectronics and Microelectronics (ICOM), 2012 International Conference on. IEEE: 372-377. (2012)

4. Hilkert, J.M. and B. Pautler. A reduced-order disturbance observer applied to inertially stabilized line-of-sight control. SPIE Defense, Security, and Sensing, International Society for Optics and Photonics, (2011).

5. Xiangyang Zhou, Hongyan Zhang, Ruixia Yu. Decoupling control for two-axis inertially stabilized platform based on an inverse system and internal model control. Mechatronics; 24(8): 1203-1213. (2014)

6. Zhuchong Lin; Kun Liu. Inertially stabilized line-of-sight control system using a magnetic bearing with vernier gimbaling capacity. Photonics Asia. International Society for Optics and Photonics: 92720Q-92720Q-11. (2014)
7. Jiancheng Fang, Chune Wang, and Tong Wen. Design and optimization of a radial hybrid magnetic bearing with separate poles for magnetically suspended inertially stabilized platform. IEEE Transactions on Magnetics; 50(5): 1-11. (2014)

8. Mu Q, Liu G, Lei X. A RBFNN-based adaptive disturbance compensation approach applied to magnetic suspension inertially stabilized platform. Mathematical Problems in Engineering, (2014).

9. Fumio Matsumura, Member, IEEE, Tom Namerikawa, Member, IEEE, Kazuhiko Hagiwara, and Masayuki Fujita, Member, IEEE. Application of gain scheduled $\mathrm{H} \infty$ robust controllers to a magnetic bearing. IEEE Transactions on Control System Technology; 4(5): 484-493(1996).

10. Zdzislaw Gosiewski, Arkadiusz Mystkowski. Robust control of active magnetic suspension:Analytical and experimental results. Mechanical Systems and Signal Processing. 22:1297-1303. (2008)

11. Shyh-Leh Chen, Member, IEEE, and Cheng-Chi Weng. Robust Control of a Voltage-Controlled Three-Pole Active Magnetic Bearing System. IEEE/ASME Transactions on Mechatronics. 15 (3): 381-388. (2010)

12. Masayuki Fujita, Toru Namerikawa, Fumio Matsumura, and Kenko Uchida. $\mu$-Synthesis of an Electromagnetic Suspension System. IEEE Transactions on Automatic Control 40(3): 530-536. (1995). 